\title{
Crecimiento económico, población, alimentos, energía y medio ambiente: la nueva agenda de APEC
}

DOI: $10.32870 /$ mycp.v4i14.147

$\mathrm{E}$ n la reunión de Osaka, Japón, (1995) del foro de Cooperación Económica de AsiaPacífico (APEC), se identificaron tres pilares para la cooperación económica de la región: liberalización del comercio, facilitación de los negocios, y la cooperación técnica y económica, (ECOTECH). El término de pilares hace referencia a los principales soportes para la discusión, los planes de acción y las grandes tareas que se pretenden realizar de manera conjunta entre las economías miembro.

En recientes reuniones una preocupación adicional ha ido tomando fuerza; Ippei Jamazawa (de la Universidad de Hitotsubashi, Tokio, Japón) la considera una de las tres grandes áreas del foro ${ }^{1}$ : la primera la integran lo que anteriormente se identificaba como los dos primeros pilares de APEC (actualmente se le conoce como la que facilita la Liberación del Comercio y las Inversiones (TILF); la segunda, y no la tercera, sería ECOTECH; finalmente, la tercera, como la identifica el autor antes mencionado, tiene que ver con cinco aspectos que están muy relacionados entre sí y que pueden llegar a convertirse en verdaderos problemas en un futuro reciente. Se trata de la relación existente entre crecimiento económico y de la población sobre la mayor demanda de alimentos y el consumo de energía, así como el efecto negativo que esto tiene sobre el medio ambiente. En la agenda de APEC estos cinco elementos son identificados como: food, energy, economy, environment y population, (FEEEP). Este tema fue propuesto inicialmente por el primer ministro Tomiichi Murayama en la reunión de líderes de Osaka de 1995; sin embargo, su origen se remonta a la propuesta de Japón formulada previamente en tres sectores conocidos con el nombre de Triple E: energía, crecimiento

* Investigador del Departamento de Estudios del Pacífico de la Universidad de Guadalajara.
Ramón Robledo Padilla* económico y medio ambiente. Posteriormente se incorporaron a estas tres áreas los temas de alimentos y población. En la declaración de Manila de 1996 la propuesta fue reiterada, y Canadá la promovió en $1997^{2}$.

Si tenemos en cuenta que el foro de APEC tiene como finalidad potenciar el crecimiento de las economías de la región, resulta evidente la pertinencia del análisis integral de las cinco variables FEEEP, así como la amenaza que éstas representan para el desarrollo sustentable en Asia-Pacífico.

Un aspecto novedoso del enfoque es la atención que se pone sobre el medio ambiente, dado el impacto negativo que el crecimiento de los cuatro elementos puede tener sobre éste.

El enfoque de sustentabilidad constituye hoy una fuente inagotable en la discusión sobre el futuro del planeta. Desde su génesis moderna en el Informe Brundtland ${ }^{3}$ de 1987 (conocido también como el informe "Nuestro Futuro Común", presentado por la Comisión Mundial Sobre el Medio Ambiente y el Desarrollo), ha servido para despertar una conciencia de alcance mundial sobre la importancia del medio ambiente, aunque todavía no muestra su utilidad para reorientar las políticas de crecimiento que deterioran constantemente el entorno ecológico.

El concepto de sustentabilidad propone satisfacer las necesidades presentes sin comprometer las expectativas de generaciones futuras ${ }^{4}$. Sin embargo, este mandato tiene que reflejar la realidad económica: cualquier acción que se emprenda para mejorar el medio ambiente natural involucra un costo, y desde el momento que no podemos hacer todo lo que deseamos, lo único que resta hacer es un balance sobre los costos que implican la preservación del 
equilibrio ecológico con respecto a los beneficios que esto nos retribuye ${ }^{5}$.

Esta postura es entendida como una visión antropocéntrica, en el sentido de que a pesar de que existe el reconocimiento del daño que ha causado el progreso económico al medio ambiente, no cuestiona el hecho de que la naturaleza esté al servicio del hombre. Confía en que la naturaleza humana, apoyada en el conocimiento científico-tecnológico, podrá revertir el daño causado al medio ambiente ${ }^{6}$.

\section{Por antinomia con la cosmovisión} antropocéntrica, el pensamiento biocéntrico considera que la raíz de la crisis del medio ambiente tiene su origen en la cultura occidental, que, con un pensamiento antropocéntrico, generó un homo sapiens conquistador y dominador de la naturaleza, que estableció con ella una relación utilitarista al entenderla como un recurso a su servicio. De esta manera, se plantea la necesidad de fundar una nueva visión del mundo, no antropocéntrica, con su correspondiente orden sociopolítico, a fin de superar el colapso ambiental y social en que nos encontraríamos en un futuro reciente?

Hasta el momento, lo que ha predominado es la visión antropocéntrica del desarrollo sustentable, aunque cada vez, y con mayor fuerza, diversos actores sociales adquieren mayor conciencia, y el debate se centra en que se requiere de la participación de un Estado más fuerte que el intervencionista del pasado. Un Estado fortalecido en su capacidad reguladora y de planificación, dejando al mercado las actividades de naturaleza estrictamente productiva o de infraestructura, y que privilegie la complementariedad entre la regulación pública y los mecanismos de mercado ${ }^{8}$.
Considerando lo anterior, el problema se debe abordar no sólo tomando en cuenta el crecimiento económico y su impacto en el deterioro del medio ambiente; sino desde la perspectiva de que el primero y el incremento en la población implican una mayor demanda de bienes (incluidos los alimentos) y servicios y, por la misma razón, un mayor consumo de energía, lo que posteriormente impactaría la relación de esos elementos con el entorno ecológico.

Uno de los enfoques más generalizados para formular la interacción entre los elementos de FEEEP, consiste en reorganizar las cinco variables por orden de causa y efecto. Esto significa examinar el impacto producido por la expansión demográfica y el crecimiento económico sobre el sector de alimentos, energía y ambiente, tal como aparece en la Declaración de los Líderes de Osaka y en el documento del presidente del Comité Económico. Por ejemplo, el proyecto sobre escenarios de las Naciones Unidas prevé que la población mundial pasará de 5,800 millones de personas en 1996 a aproximadamente 10 mil millones para el año 2050 , lo que representa un crecimiento de $72 \%$. En el caso de los miembro de APEC, el crecimiento será más moderado (36\%), lo que incrementará su población de 2,200 millones de habitantes en 1996 a 3 mil millones en 2050. Si se multiplica este aumento de la población por una tasa de crecimiento adecuada del PIB (una extrapolación modificada de la tendencia pasada) tendremos un aumento futuro de las demandas de alimentos, de energía y de contaminación. Si la oferta de alimentos y de energía requerida no puede cubrir el aumento proyectado en la demanda, el escenario esperado no se produciría. De igual forma, se deben analizar las medidas necesarias para evitar la contaminación ambiental prevista y el costo que 
esto implicaría. En el caso de que el costo fuera muy elevado o de que no pudiera evitarse la contaminación, se registraría el escenario original y una gran degradación de los estándares ambientales. Si sólo aumentara la población, la caída del crecimiento económico total reduciría el nivel en la calidad de vida, por la disminución en el ingreso per cápita. Con todo esto, el escenario de un crecimiento importante en las economías de APEC no será sostenible a largo plazo si no se toman las medidas precautorias con base en un análisis cuidadoso de los problemas a los que se tendrán que enfrentar no sólo las economías de APEC, sino también la economía global ${ }^{9}$. Teniendo en cuenta estas consideraciones, en las discusiones realizadas en 1997 en Canadá, el análisis sobre FEEEP se dividió en tres áreas: alimentos, energía y medio ambiente. En lo que se refiere a los alimentos, el reporte señala que la región en su conjunto no ha experimentado problemas de hambruna o carencia de alimentos en los pasados treinta años; al mismo tiempo, se indica que los precios de los granos en términos reales han ido a la baja. Así, hay optimismo, dado que las tasas de crecimiento poblacional en APEC, en su conjunto, han estado declinando, lo cual se refleja en un incremento en los niveles de ingreso y una baja en la fertilidad notoria por los cambios en la estructura poblacional de edades. Además, la comunidad mundial se encuentra en el umbral para construir investigaciones biotecnológicas básicas que logren resultados prácticos ${ }^{10}$.

En el área de energía, las economías miembro de APEC absorben aproximadamente la mitad de la energía consumida en el mundo, y se espera un crecimiento en el consumo de 2.2 $\%$ anual. Por esta razón, se planteó que era necesario coordinar esfuerzos con los gobiernos de la región, en asociación con los empresarios y el público en general, para facilitar la construcción de una infraestructura de energía sana, en términos ambientales ${ }^{11}$.

En lo que concierne al medio ambiente, se planteó promover una producción más limpia en la industria por medio de la identificación y la expansión del uso de métodos de producción más adecuados, y del establecimiento de una agenda para la difusión tecnológica, con particular atención para las pequeñas y medianas empresas. En especial, se identificó al sector agrícola como una importante área para la aplicación de estas medidas ${ }^{12}$.

Finalmente, en las discusiones llevadas a cabo en 1997, se reconoció que los resultados en el área de FEEEP podrían ser positivos o negativos, dependiendo de las decisiones y del comportamiento de los productores y consumidores de muchos grupos dentro de nuestras sociedades, incluyendo miembros del gobierno, autoridades locales, líderes de negocios, científicos y, finalmente, los individuos y comunidades que viven en áreas rurales y urbanas. Así pues, existió consenso en que la difícil tarea para lograr avances en FEEEP, requiere de compromisos complementarios entre el mercado, el gobierno y la comunidad en general $^{13}$.

Dado que la interacción de los cinco elementos mencionados no es un problema exclusivo de APEC, el análisis de FEEEP se tendría que hacer en tres dimensiones espaciales: la de un país en particular, la región de APEC y la del mundo.

En este sentido, es evidente que las cinco variables exceden los límites de los países en particular. Un ejemplo lo constituye Japón; tanto su crecimiento como sus condiciones de vida han excedido su autoabastecimiento de alimentos, energía y ambiente. El crecimiento aponés ha dependido considerablemente del comercio y la inversión extranjera. La presión que existe sobre la emigración de mano de obra es tan fuerte que ningún país puede mostrarse indiferente ante la explosión demográfica en los países vecinos. Por otro lado, existen algunos aspectos de la contaminación ambiental, como por ejemplo la lluvia ácida y algunos desechos industriales, que podrían ser confinados a las subregiones del noreste asiático o sudeste asiático. Sin embargo, es posible circunscribir una mayor proporción del comercio de alimentos, de energía y de la emigración de mano de obra a la región de Asia-Pacífico. Es decir, la demanda 
regional se cubre con la oferta regional a través del comercio intrarregional. En el régimen de economía de mercado abierto que adoptan las economías de APEC, cualquier déficit significativo de alimentos y energía en la economía de un país miembro es transferido a otros miembros en forma de precios más elevados y de ajustes en toda la región ${ }^{14}$. Quizá, el reto principal que se deben plantear hoy en día las economías de APEC y del mundo en general, no es cuánto crecer, sino cómo crecer.

\section{Notas}

1 http://www.asiayargentina.com/Sela-2.PDF p. 14.

2 Ibidem.

3 En la definición de desarrollo sustentable planteada en el Informe Brundtland de1987, se señala que el crecimiento económico y el uso racional de los recursos naturales y el medio ambiente están vinculados.

4 Torres Torres, Felipe pp. 13 y 14. (ver p. sig.)

5 García Páez, Benjamín p. 111. (ver p. sig.)

6 Estensoro Saavedra, Jaime Fernando p. 146. (ver p. sig.)

7 Ibid.p. 140.

8 Ibid.p. 148.

9 Op.cit. http://www.asiayargentina.com/Sela-2.PDF p. 21.

10 http://intlag.tamu.edu/cuiriak.htm p. 3.

11 Idem. pp. 4 y 5.

12 Idem p. 6.

13 Idem p. 8.

$14 \mathrm{Op}$. cit. http://www.asiayargentina.com/Sela-2.PDF p. 22.

\section{Fuentes}

Estensoro Saavedra, Jaime Fernando, "Proyección ideológica del debate en torno al medio ambiente", Ciencia Política, Vol. XXI, No1, 2001, pp. 135-153.

García Páez, Benjamín, Economía ambiental, Universidad Nacional Autónoma de México, Facultad de Economía, México 2000.

Torres Torres, Felipe y Trápaga Delfín, Yolanda, La agricultura orgánica: una alternativa para la economía campesina de la globalización, Universidad Nacional Autónoma de México, México 1997.

http://intlag.tamu.edu/cuiriak.htm

http://www.asiayargentina.com/Sela-2.PDF. 19: 